\title{
Observing symmetry-broken optimal paths of stationary Kardar-Parisi-Zhang interface via a large-deviation sampling of directed polymers in random media
}

\author{
Alexander K. Hartmann, ${ }^{1, *}$ Baruch Meerson, ${ }^{2}, \dagger$ and Pavel Sasorov ${ }^{3,4, \ddagger}$ \\ ${ }^{1}$ Institut für Physik, Universitåt Oldenburg - 26111 Oldenburg, Germany \\ ${ }^{2}$ Racah Institute of Physics, Hebrew University of Jerusalem, Jerusalem 91904, Israel \\ ${ }^{3}$ Institute of Physics CAS - ELI Beamlines, 18221 Prague, Czech Republic \\ ${ }^{4}$ Keldysh Institute of Applied Mathematics, Moscow 125047, Russia
}

\begin{abstract}
Consider the short-time probability distribution $\mathcal{P}(H, t)$ of the one-point interface height difference $h(x=0, \tau=t)-h(x=0, \tau=0)=H$ of the stationary interface $h(x, \tau)$ described by the KardarParisi-Zhang equation. It was previously shown that the optimal path - the most probable history of the interface $h(x, \tau)$ which dominates the upper tail of $\mathcal{P}(H, t)$ - is described by any of two ramplike structures of $h(x, \tau)$ traveling either to the left, or to the right. These two solutions emerge, at a critical value of $H$, via a spontaneous breaking of the mirror symmetry $x \leftrightarrow-x$ of the optimal path, and this symmetry breaking is responsible for a second-order dynamical phase transition in the system. We simulate the interface configurations numerically by employing a large-deviation Monte Carlo sampling algorithm in conjunction with the mapping between the KPZ interface and the directed polymer in a random potential at high temperature. This allows us to observe the optimal paths, which determine each of the two tails of $\mathcal{P}(H, t)$, down to probability densities as small as $10^{-500}$. At short times we observe mirror-symmetry-broken traveling optimal paths for the upper tail, and a single mirror-symmetric path for the lower tail, in good quantitative agreement with analytical predictions. At long times, even at moderate values of $H$, where the optimal fluctuation method is not supposed to apply, we still observe two well-defined dominating paths. Each of them violates the mirror symmetry $x \leftrightarrow-x$ and is a mirror image of the other.
\end{abstract}

\section{INTRODUCTION}

Large deviations of fluctuating quantities in stochastic macroscopic systems out of equilibrium continue to attract much interest from the statistical mechanics community. Of special interest are situations where the largedeviation functions exhibit singularities, see Refs. [1-5] for reviews. Among these there are singularities which can be classified as dynamical phase transitions (DPTs) [6-9], because the pertinent large-deviation functions involve time $t$, and $t \rightarrow \infty$. Here we consider the celebrated Kardar-Parisi-Zhang (KPZ) equation [10] in 1+1 dimension,

$$
\partial_{\tau} h=\nu \partial_{x}^{2} h+(\lambda / 2)\left(\partial_{x} h\right)^{2}+\sqrt{D} \xi(x, \tau) .
$$

It has been shown recently $[11,12]$ that the KPZ equation also exhibits a DPT, but in the opposite limit of $t \rightarrow 0$. This DPT, and breaking of the mirror symmetry $x \leftrightarrow-x$ of the optimal paths of the interface, i.e. the interface height histories $h(x, t)$ which contribute most to rareevent statistics which we will specify shortly will be the focus of attention of this work.

The KPZ equation (1) describes the stochastic dynamics of the height $h(x, \tau)$ at point $x$ and at time $\tau$ of an interface without overhangs, driven by a delta-correlated

\footnotetext{
* a.hartmann@uni-oldenburg.de

$\dagger$ meerson@mail.huji.ac.il

‡ pavel.sasorov@gmail.com
}

Gaussian noise $\sqrt{D} \xi(x, \tau)$ with zero mean:

$$
\begin{aligned}
\langle\xi(x, \tau)\rangle & =0, \\
\left\langle\xi\left(x_{1}, \tau_{1}\right) \xi\left(x_{2}, \tau_{2}\right)\right\rangle & =\delta\left(x_{1}-x_{2}\right) \delta\left(\tau_{1}-\tau_{2}\right) .
\end{aligned}
$$

In Eq. (1) $\nu>0$ is the diffusion constant, and the nonlinearity coefficient $\lambda$ can be set to be positive (or negative) without loss of generality.

The fluctuating quantity of our interest here is the interface height difference,

$$
h(x=0, \tau=t)-h(x=0, \tau=0)=H,
$$

at a specified point $x=0$ at time $t$ [13]. The complete statistics of the height difference is encoded in the probability distribution $\mathcal{P}(H, t)$ which has been the focus of multiple recent studies, see Refs. [14-16] for reviews. Note that the distribution $\mathcal{P}(H, t)$ depends on the initial condition $h(x, \tau=0)$. Commonly studied are the sharp-wedge (also called droplet), flat and stationary initial conditions [14-16].

We will be mostly interested in the short-time regime, when the observation time $t$ is much smaller than the characteristic nonlinear time of the KPZ equation $t_{\mathrm{NL}}=$ $\nu^{5} /\left(D^{2} \lambda^{4}\right)$. At short times typical fluctuations of the KPZ interface, which corresponds to the body of $\mathcal{P}(H, t)$, are still Gaussian. However, the KPZ nonlinearity is already fully manifest in the tails of $\mathcal{P}(H, t)$. The DPT $[11,12]$ occurs, in the short-time regime, for the stationary initial condition, where it is assumed that the interface has evolved for a long time prior to $\tau=0$. For the stationary interface, initial configurations $h(x, \tau=0)$ are sampled from a statistical ensemble of random realiza- 
tions of a two-sided Brownian motion:

$$
h(x, \tau=0)=\sqrt{\frac{D}{2 \nu}} B(x) .
$$

Here $B(x)$ is the two-sided Wiener process with diffusion constant $1 / 2$, so that $\left\langle B^{2}(x)\right\rangle=|x|[17]$. The shorttime probability distribution $\mathcal{P}(H, t)$ scales, up to a preexponential factor, as $[11,18]$

$$
-\ln \mathcal{P}(H, t) \simeq \frac{\nu^{5 / 2}}{D \lambda^{2} \sqrt{t}} s\left(\frac{|\lambda| H}{\nu}\right),
$$

where the scale function $s(\ldots)$ is given by the (rescaled) effective action along the optimal path of the interface, as we explain below. The function

$$
S(H)=-\lim _{t \rightarrow 0} \sqrt{t} \ln \mathcal{P}(H, t)=\frac{\nu^{5 / 2}}{D \lambda^{2}} s\left(\frac{|\lambda| H}{\nu}\right)
$$

is the short-time large deviation function. Remarkably, $S(H)$ exhibits a second-order DPT (that is a jump in the second derivative $d^{2} S / d H^{2}$ ) at a critical value of $H$ such that $\lambda H_{c} / \nu=3.7063 \ldots$ The DPT originates from a spontaneous breaking of mirror symmetry of the optimal path which we will discuss shortly. A rather complete analogy of this DPT with the classical mean-field second-order transition in equilibrium was established in Ref. [12], where a proper phase order parameter was identified, and an effective Landau theory was developed.

Ref. [11] also determined the large- $|H|$ asymptotics of $S(H)$

$$
S(H) \simeq \begin{cases}\frac{4 \sqrt{2} \nu}{3 D|\lambda|^{1 / 2}}|H|^{3 / 2}, & \lambda H \rightarrow+\infty, \\ \frac{4 \sqrt{2|\lambda|}}{15 \pi D}|H|^{5 / 2}, & \lambda H \rightarrow-\infty,\end{cases}
$$

which correspond to the (stretched-exponential) upper and lower tails of the distribution $\mathcal{P}(H, t)$.

In this paper we report, to our knowledge first, observations of the optimal paths of the KPZ interface, which determine the distribution tails (6) and (7) for the stationary interface, in numerical simulations. Here is some background information. The results of Refs. [11, 12] were obtained by the optimal fluctuation method (OFM) (see also Ref. [19]). The OFM - by now a standard asymptotic tool of theoretical physics - relies on a saddlepoint evaluation of the path integral of the KPZ equation (1), conditioned on reaching a specified height $H$ at short times (or reaching a sufficiently large height $H$ at any time). This procedure brings about a classical field theory, formulated as a conditional variational problem. The solution of this problem, obeying the proper initial and boundary conditions, is called the optimal path of the conditioned process. It represents a time-dependent deterministic field which describes the most probable time history of the system, dominating the contribution of different paths to the statistics of interest. Once the optimal path is determined, the action along it (plus the "cost" of the optimal initial condition, if this additional optimization is present) yields the scale function $s(\ldots)$ which enters Eq. (4). The OFM has been extensively used for studying the single-point height statistics of the $\mathrm{KPZ}$ equation in different settings and dimensions and for different initial conditions [20-33]. Until very recently, exact analytical solutions of the OFM equations for the KPZ interface were only possible when using additional small parameters, such as a very large or very small $|H|$. But in Refs. [34, 35] the complete short-time large-deviation functions for $\mathcal{P}(H, t)$ have been obtained analytically for the droplet, flat and stationary initial conditions by masterfully exploiting exact integrability of the OFM equations pointed out in Ref. [11].

Without losing generality, we will suppose that $\lambda>0$. Crucially, at $H>H_{c}$ the OFM predicts the existence of two optimal paths of the interface, each leading to the same scaling function $s(\ldots)[11,12]$. The two optimal solutions bifurcate from a single solution at the critical point $H=H_{c}$ via a spontaneous breaking of the mirror symmetry - the spatial reflection symmetry $x \leftrightarrow-x$, leading to the second-order DPT in $s(\ldots)$. Each of the two optimal solutions is a mirror-symmetric "twin" of the other with respect to $x \leftrightarrow-x$. At large positive $H$, each of the two solutions has the form of a ramp-like traveling structure of $h(x, \tau)$ or, equivalently, a shock-antishock pair of the interface slope $V(x, \tau)=\partial_{x} h(x, \tau)$. For $-\infty<$ $H<H_{c}$, that is below the transition, the optimal path is unique and mirror symmetric with respect to $x=0$.

Besides their role in the calculation of large-deviation functions, optimal paths provide a valuable insight into the physics of large deviations, and this insight is inaccessible by other methods. However, a direct observation of optimal paths in conventional numerical simulations (and of course in experiments) is often difficult due to the very low probability of the large deviations in question [36]. Fortunately, this difficulty can be overcome by using large-deviation algorithms, e.g. based on importance sampling approaches, as was recently shown for the KPZ equation in Refs. [37-39]. In these works a mapping from the KPZ equation to the (discrete) directed polymer in a random potential at high temperature was employed, and Monte-Carlo simulations with an importance sampling algorithm were performed. As a result, the tails of the height distribution of the KPZ interface were measured, for different initial conditions and at different times, down to probabilities as small as $10^{-1000}[37,39]$. In particular, Ref. [39] measured the whole short-time distribution of $\mathcal{P}(H, t)$ for the stationary interface and, in particular, verified the asymptotics (6) and (7). In Ref. [38] the interface configurations at $\tau=t / 2$, corresponding to the height distribution tails at $\tau=t$, were observed for the droplet initial condition. The observed configurations turned out to be in good agreement with those predicted from the OFM [24].

Here we employ the same simulation strategy as in Refs. [37-39]. Our primary goal is to observe the optimal paths of the stationary KPZ interface which correspond 
to the distribution tails (6) and (7). Apart from the short-time regime, where the OFM is well known to apply, we also consider a long-time regime where the mere existence of optimal paths has not been established.

Here is a layout of the remainder of the paper. In Sec. II we extend and elaborate on theoretical predictions of Refs. [11, 12] for the optimal paths. In Sec. III we present our simulation strategy. In Sec. IV we compare the simulation results with theory at short times, where the OFM is expected to apply $[11,12]$. In Sec. V we present the results of our simulations in a long-time regime. This includes probing a range of moderate (not too large) values of $H$, where one does not expect OFM to be applicable. In Sec. VI we briefly summarize and discuss the main results of this work.

\section{OPTIMAL PATHS: THEORETICAL PREDICTIONS}

Here we present and extend the theoretical predictions of Refs. [11, 12]. To be in line with the notation of Refs. $[11,12]$, we will temporarily switch to the convention $\lambda<0$. The final results of this section will be presented in a fully dimensional form with an arbitrary sign of $\lambda$. Introduce rescaled variables $\tau / t \rightarrow \tau, x / \sqrt{\nu t} \rightarrow x$ and $|\lambda| h / \nu \rightarrow h$. Then Eq. (1) becomes dimensionless:

$$
\partial_{\tau} h=\partial_{x}^{2} h-\frac{1}{2}\left(\partial_{x} h\right)^{2}+\sqrt{\epsilon} \xi(x, \tau)
$$

where

$$
\epsilon=\frac{D \lambda^{2} \sqrt{t}}{\nu^{5 / 2}}=\left(\frac{t}{t_{\mathrm{NL}}}\right)^{1 / 2}
$$

is the rescaled noise magnitude. We choose the reference frame of the interface position so that

$$
h(x=0, \tau=0)=0
$$

and condition the rescaled stochastic process $h(x, \tau)$ on the equality

$$
h(x=0, \tau=1)=H,
$$

where $H$ is rescaled by $\nu /|\lambda|$. Formally, the OFM demands that $\epsilon \rightarrow 0$, but later on we will also consider regimes of finite and even large $\epsilon$. The saddle-point procedure brings about a minimization problem for an effective action functional $s[h(x, \tau)]$. For the stationary interface this functional includes two terms [11]:

$$
s[h(x, \tau)]=s_{\mathrm{dyn}}[h(x, \tau)]+s_{\mathrm{in}}[h(x, 0)],
$$

where

$$
s_{\mathrm{dyn}}[h(x, \tau)]=\frac{1}{2} \int_{0}^{1} d \tau \int_{-\infty}^{\infty} d x\left[\partial_{\tau} h-\partial_{x}^{2} h+\frac{1}{2}\left(\partial_{x} h\right)^{2}\right]^{2}
$$

is the dynamical contribution, whereas

$$
s_{\text {in }}[h(x, 0)]=\left.\int_{-\infty}^{\infty} d x\left(\partial_{x} h\right)^{2}\right|_{\tau=0}
$$

is the "cost" of the initial height profile (3) [11]. The ensuing Euler-Lagrange equation can be recast in the Hamiltonian form

$$
\begin{aligned}
& \partial_{\tau} h=\partial_{x}^{2} h-\frac{1}{2}\left(\partial_{x} h\right)^{2}+\rho, \\
& \partial_{\tau} \rho=-\partial_{x}^{2} \rho-\partial_{x}\left(\rho \partial_{x} h\right),
\end{aligned}
$$

where $\rho(x, \tau)$, the optimal realization of the (rescaled) noise $\xi(x, \tau)$, plays the role of the "momentum density" field, which is canonically conjugate to the "coordinate density", which is the optimal path $h(x, \tau)$ itself.

The minimization over $h(x, \tau=0)$ yields an initial condition in the form of a relation between a priori unknown $h(x, 0)$ and $\rho(x, 0)[11]$ :

$$
\rho(x, \tau=0)+2 \partial_{x}^{2} h(x, \tau=0)=\Lambda \delta(x) .
$$

This condition is specific to the stationary interface. The one-point condition (11) leads to the singular condition

$$
\rho(x, \tau=1)=\Lambda \delta(x),
$$

where $\Lambda$, which plays the role of a Lagrangian multiplier, is to be ultimately expressed through $H$.

Once the optimal path, including the optimal initial height profile, is determined, one can evaluate the rescaled total action (12), and obtain the scaling function $s=s(H)$ which enters Eqs. (4) and (5).

In Refs. [11] and [12] asymptotic solutions of the OFM problem (15)-(18) were obtained in the two limits, corresponding to the upper and lower tails of $\mathcal{P}$ and described by Eqs. (6) and (7), respectively. We now extend and elaborate on the pertinent optimal solutions for $h(x, t)$, to be compared with our simulations in Sec. IV.

\section{A. Optimal path for the upper tail $\lambda H>0$}

Here we use the dimensional variables. At supercritical values of $H$ there are two mirror-symmetry broken optimal paths [11]. In the $\lambda H \rightarrow+\infty$ tail, each of them represents a simple ramp-like structure for $h(x, \tau)$ [or, equivalently, a shock-antishock pair of the interface slope $\left.V(x, \tau)=\partial_{x} h(x, \tau)\right]$, traveling to the left or to the right. In terms of $h(x, \tau)$ the left-traveling ramp has the form [11]:

$$
\frac{h(x, \tau)}{H} \simeq \begin{cases}1, & x \geq \ell(1-\mathfrak{t}) \\ \frac{x}{\ell}+\mathfrak{t}, & -\ell \mathfrak{t} \leq x \leq \ell(1-\mathfrak{t}), \\ 0, & x \leq-\ell \mathfrak{t} .\end{cases}
$$

where $\mathfrak{t}=\tau / t$ and

$$
\ell=\ell(H, t)=\left(\frac{|\lambda H| t}{2}\right)^{1 / 2}
$$


The right-traveling ramp solution can be obtained from Eqs. (19)-(21) by replacing $x$ by $-x$. Snapshots of the right- and left-traveling solutions for $h(x, \tau)$ are depicted in Fig. 1.
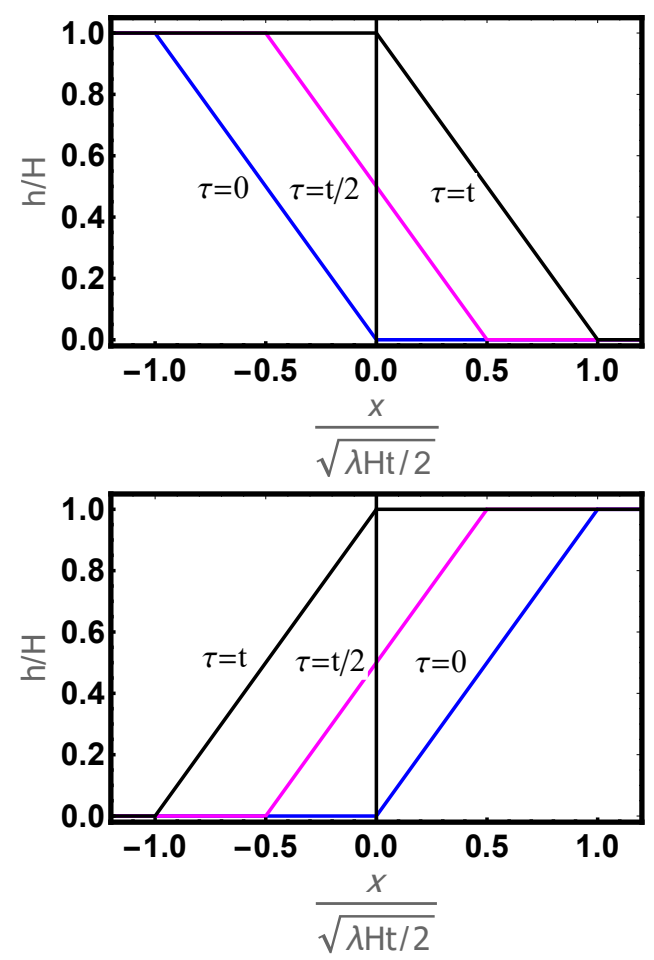

FIG. 1. Two mirror-symmetry broken optimal paths corresponding to the upper tail, $\lambda H>0$, of the height distribution $\mathcal{P}(H, t)$, see Eq. (6). Shown is the leading-order prediction of Ref. [11] for $h(x, \tau)$ as described by Eqs. (19)-(21) for the ramp traveling to the left (the right panel), and its mirror reflection with respect to $x=0$ for the ramp traveling to the right (the left panel).

\section{B. Optimal path for the lower tail $\lambda H<0$}

At $\lambda H \rightarrow-\infty$ the leading-order solution is very different $[11,12,23,24]$. In the "pressure-dominated" region the solution describes an ideal hydrodynamic flow of an effective gas with density $\rho(x, \tau)$, velocity $V(x, \tau)=$ $\partial_{x} h(x, \tau)$ and a negative pressure $-(1 / 2) \rho^{2}(x, \tau)$. There are also a region of a zero-pressure hydrodynamic flow (also called the Hopf flow) [12] and a trivial "static region" where $h(x, \tau)=H / 2=$ const.

The results of Refs. [11, 12, 23, 24] were presented in terms of $V(x, \tau)$ rather than $h(x, \tau)$. Here we calculated $h(x, \tau)$ by integrating $V(x, \tau)$ over $x$. After some algebra we obtained, in the three regions,

$$
\frac{h(x, \tau)}{H} \simeq \begin{cases}\mathfrak{h}_{g}\left(\frac{\pi x}{2 \ell}, \mathfrak{t}\right)+\frac{1}{2}, & \frac{\pi|x|}{2}<\frac{\ell}{1+w(\mathfrak{t})^{2}}, \\ \mathfrak{h}_{v}\left(\frac{\pi|x|}{2 \ell}, \mathfrak{t}\right)+\frac{1}{2}, & \frac{\ell}{1+w(\mathfrak{t})^{2}}<\frac{\pi|x|}{2}<\ell \\ \frac{1}{2}, & \frac{\pi|x|}{2}>\ell .\end{cases}
$$

where $\ell$ is defined in Eq. (22), and we have introduced three functions: $w, \mathfrak{h}_{g}$, and $\mathfrak{h}_{v}$. The function $w(\mathfrak{t})$ is the inverse of the function

$$
\mathfrak{t}=\frac{1}{2}+\frac{w}{\pi\left(1+w^{2}\right)}+\frac{1}{\pi} \arctan w,
$$

the function $\mathfrak{h}_{g}(\chi, \mathfrak{t})$ is defined explicitly:

$$
\mathfrak{h}_{g}(\chi, \mathfrak{t})=\frac{1}{\pi} \arctan w(\mathfrak{t})-\frac{1}{\pi} w(\mathfrak{t})\left[1+w^{2}(\mathfrak{t})\right] \chi^{2},
$$

and the function $\mathfrak{h}_{v}(\chi, \mathfrak{t})$ is defined in a parametric form:

$$
\begin{aligned}
\mathfrak{h}_{v}(z, \mathfrak{t}) & =u^{2}\left(\mathfrak{t}-\frac{1}{2}\right)+\frac{1}{\pi}\left[u+\left(u^{2}-1\right) \arctan u\right], \\
z & =\pi u\left(\mathfrak{t}-\frac{1}{2}\right)+1+u \arctan u
\end{aligned}
$$

where the domain of $u$ in Eqs. (28)-(29) is defined by the double inequality

$$
-w(\mathfrak{t})\left(\mathfrak{t}-\frac{1}{2}\right)<u\left(\mathfrak{t}-\frac{1}{2}\right)<0 .
$$

Overall, the optimal $h$-profiles (23)-(25) as a function of $x$ at different times $0 \leq \tau \leq t$ are depicted in Fig. 2 . Notice that the optimal initial height profile has a plateau at $h=H / 2$ at large $|x|$. This property holds exactly for all subcritical values of $H:-\infty<\lambda H<|\lambda| H_{c}$, where the mirror symmetry of the optimal path is preserved.

\section{SIMULATIONS}

\section{A. Directed polymer mapping}

Let us recall the lattice version of the mapping between the KPZ height $h(x, t)$ and the free energy of a directed polymer in a two-dimensional random potential at high temperature $T$ [40]. To follow the evolution of the height profiles in time, it is convenient to work with a righttriangular domain, defined on a half square lattice with side length $L$ as shown in Fig. 3. The lattice is indexed by points $(x, \tau)$ with $\tau=0,1, \ldots, t(t=2 L)$ playing the role of a time and $x=-x_{\max },-x_{\max }+1, \ldots, x_{\max }-1, x_{\max }$ where $x_{\max }=(t-\tau) / 2$. We consider all directed polymers which start at a lattice point on the hypotenuse $(x, 0)$ and end at the apex $(0,2 L)$. The random value of the potential $V$ at each lattice point is normally distributed with zero mean and unit variance. The partition 


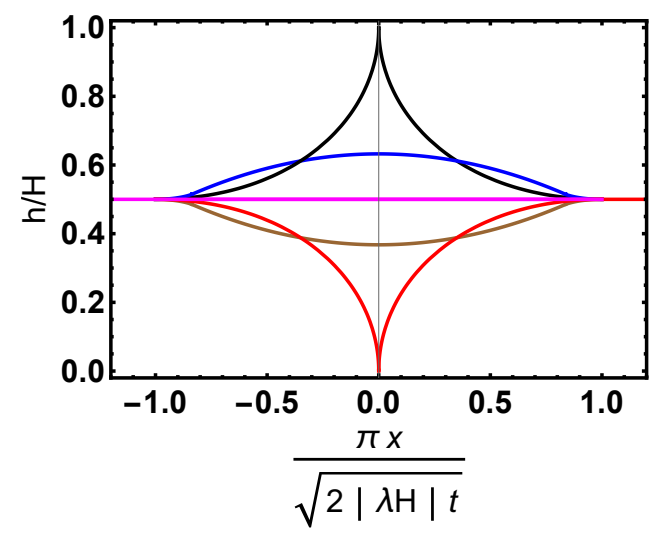

FIG. 2. The mirror-symmetric optimal paths corresponding to the lower tail, $\lambda H<0$, of the height distribution $\mathcal{P}(H, t)$, see Eq. (7). Shown are the leading-order theory prediction for $h(x, \tau)$ vs $x$ as described by Eqs. (23)-(25) at rescaled times $\mathfrak{t}=\tau / t=0$ (black), 0.25 (blue), 0.5 (magenta), 0.75 (brown), and 1 (red).

function $Z(x, \tau)$ of a given realization of the potential obeys the exact recursive equation [40]

$Z(x, \tau+1)=\left[Z\left(x-\frac{1}{2}, \tau\right)+Z\left(x+\frac{1}{2}, \tau\right)\right] e^{-\frac{V(x, \tau+1)}{T}}$,

where $\left\langle V(x, \tau) V\left(x^{\prime}, \tau^{\prime}\right)\right\rangle=\delta_{x x^{\prime}} \delta_{\tau \tau^{\prime}}$, and $\delta_{x x^{\prime}}$ and $\delta_{\tau \tau^{\prime}}$ are Kronecker deltas.

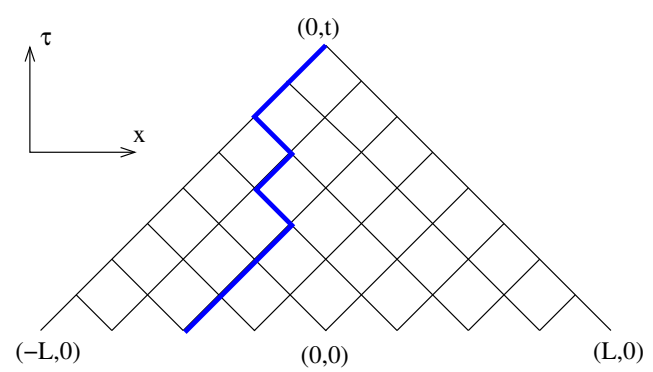

FIG. 3. A lattice realization of the directed polymer (see the text). $t=2 L$.

Let us introduce $Z^{*}(x, \tau)=2^{-\tau} Z(x, \tau)$. To obtain the mapping from the discrete Eq. (31) to the continuous KPZ equation (1) we Taylor-expand all the discrete quantities in Eq. (31), including $\exp (-V / T)$. For example,

$Z^{*}\left(x-\frac{1}{2}, \tau\right)=Z^{*}(x, \tau)-\frac{1}{2} \partial_{x} Z^{*}(x, \tau)+\frac{1}{8} \partial_{x}^{2} Z^{*}(x, \tau)+\ldots$

To justify a truncation of this expansion we must demand that $Z^{*}$ varies slowly on the scale of the lattice constant both in space and in time: $\left|Z^{*}(x, \tau+1)-Z^{*}(x, \tau)\right| \ll$ $Z^{*}(x, \tau)$ and $\left|Z^{*}(x+1, \tau)-Z^{*}(x, \tau)\right| \ll Z^{*}(x, \tau)$. This leads to the necessary conditions $L \gg 1$ and $|H| \ll L$. As we are studying large deviations, we consider $|H| \gg 1$.
Finally, the slow variation of the potential $V$ necessitates the condition $T \gg 1$. Overall, these necessary conditions can be rewritten as a strong double inequality

$$
1 \ll|H| \ll L
$$

Under these conditions we can approximate the discrete equation (31) by the continuous stochastic heat equation

$$
\partial_{\tau} Z^{*}=\frac{1}{8} \partial_{x}^{2} Z^{*}-\frac{\xi}{T} Z^{*}
$$

The (multiplicative) noise term $\xi$ is now continuous and delta-correlated in $x$ and $\tau$, and the mapping to the KPZ equation is given by the Cole-Hopf transformation

$$
h(x, \tau)=\ln \left[Z^{*}(x, \tau) /\left\langle Z^{*}(0, \tau)\right\rangle\right],
$$

where

$$
\lambda=1 / 4, \quad \nu=1 / 8, \quad D=T^{-2} \quad \text { and } \quad t=2 L .
$$

In particular, the height difference is given by

$$
H=h(0,2 L)=\ln \left[Z^{*}(0,2 L) /\left\langle Z^{*}(0,2 L)\right\rangle\right] .
$$

The characteristic nonlinear time of the KPZ equation is $t_{\mathrm{NL}}=\nu^{5} /\left(D^{2} \lambda^{4}\right)=T^{4} / 2^{7}$, and $\epsilon=16 \sqrt{L} / T^{2}$.

The Brownian initial condition is specified by

$$
Z(x, 0)=e^{-[V(x, 0)+2 R(x)] / T},
$$

where $R(x)=\sum_{0}^{x} \eta(x)$ describes a random walk starting at the origin $(0,0)$ and arriving at the site $(x, 0)$ on the hypothenuse, where $-L \leq x \leq L$. The random walk increments $\eta(x)$ are independent and normally distributed random numbers with zero mean and variance 1 . At large values of $|x|$, the random walk $R(x)$ approaches the Brownian motion $B(x)$, and the coefficient $2 / T$ in the exponent of Eq. (38) corresponds to the coefficient $D /(2 \nu)$ in Eq. (3).

\section{B. Importance sampling algorithm}

All measurable quantities, such as the height difference $H$, depend on the set of calculated partition function values $\{Z(x, \tau)\}$. Furthermore, the partition function values depend through Eqs. (31) and (38) deterministically on the realization $(V, \eta)$ of the quenched randomness, with $V$ being the set of $(L+1)(2 L+$ 1) potential values $\{V(-L, 0), V(-L+1,0), \ldots, V(L-$ $1,0), V(L, 0), V\left(-L+\frac{1}{2}, 1\right), V\left(-L+\frac{3}{2}, 1\right), \ldots, V\left(-\frac{1}{2}, 2 L-\right.$ 1), $\left.V\left(\frac{1}{2}, 2 L-1\right), V(0,2 L)\right\}$ and $\eta$ being the set of $2 L+1$ random walk increments $\{\eta(-L), \eta(-L+1), \ldots, \eta(L-$ $1), \eta(L)\}$. As we already mentioned, all these random values are independent and normally distributed with zero mean and variance 1 .

A straightforward way to sample measurable quantities would be to generate independent realizations $(V, \eta)$ 
of the disorder, to calculate the partition function for each realization and obtain the desired quantity. In this way estimates for expectation values can be obtained by averages, possibly conditioned on certain values of a second observable, like $H$. Estimates for distributions are obtained from histograms.

This procedure, however, would generate typical values with respect to all quantities, in particular typical values with respect to $H$. In the present study we are rather interested in investigating the behavior of the system for very rare values of $H$. Thus, we have to be able to address the underlying distribution $\mathcal{P}(H)$ over a large part of the support, in particular the low-probability tails.

To achieve this, we use a biased distribution [41] of the randomness by modifying the original quenched distribution weight [42], which is a product of independent Gaussians, by an additional exponential Boltzmann factor $e^{-H / \Theta}$, where $\Theta$ is an adjustable temperature-like parameter, allowing us to address different regions of values of $H$. When $\Theta \rightarrow \pm \infty$, we restore the original unbiased distribution. For $\Theta \rightarrow 0^{+}$, one will focus the sampling on large negative values of $H$, while for $\Theta \rightarrow 0^{-}$the sampling will be in the region of large positive values of $H$. The fundamental idea of this large-deviation approach is versatile, and it has been applied to various problems, e.g., to study large-deviation properties of random graphs $[43,44]$, of random walks $[45,46]$ of energy grids [47, 48], of biological sequence alignments [42, 49], of nonequilibrium work distributions [50] and of traffic models [51].

Note that any obtained statistics will initially follow the biased weight, but this can be easily corrected by reweighting with the inverse weight, i.e. $e^{+H / \Theta}$, and proper normalization [42]. This reweighting is important if one is interested in actually measuring $\mathcal{P}(H)$. In the present case, where we only measure some other quantities, conditioned on certain, e.g., extreme values of $H$, the reweighting is unnecessary. Thus, we only have to be able to sample with respect to selected values of $H$, which is achieved by the bias.

In the present case, the biased sampling of the disorder realizations $(V, \eta)$ cannot be performed directly. Therefore we applied Markov-chain Monte Carlo (MC) simulations [52], where a configuration of the Markov chain at step $s$ is given by a disorder realization $(V, \eta)^{(s)}$. As explained above, the corresponding height depends deterministically on the disorder realizations, i.e. $H^{(s)}=$ $H\left[(V, \eta)^{(s)}\right]$. Following the Metropolis-Hastings algorithm, in each step some of the entries of $(V, \eta)^{(s)}$ are redrawn according to the underlying Gaussian distribution, leading to a trial configuration $(V, \eta)^{\prime}$ which corresponds to a value $H^{\prime}$. This value has to be obtained in each MC step by a full calculation of the partition function using Eqs. (31) and (38) and finally evaluating $H^{\prime}$ with Eq. (37). To obtain a sampling according to the biased distribution, the trial configuration is accepted, i.e. $(V, \eta)^{(s+1)}=(V, \eta)^{\prime}$, with the usual Metropolis probability $p_{\text {acc }}=\min \left\{1, e^{-\Delta H / \Theta}\right\}$ depending on the change $\Delta H=H^{\prime}-H^{(s)}$. Otherwise the trial configuration is rejected, i.e. $(V, \eta)^{(s+1)}=(V, \eta)^{(s)}$. As usual, one has to make the MC simulations long enough to achieve equilibration and avoid correlated configurations. For more details on the implementation of the large-deviation algorithm for the KPZ model, including the choice of the values for $\Theta$, the combination of the results obtained for different values of $\Theta$, the use of the parallel tempering approach, and the parallelization using Message Passing Interface, see Refs. [37, 39].

The simulations where performed for the directed polymer model with length $t=2 L=128$ and two values of temperature: $T=2$ and $T=8$. We performed a parallel tempering simulation for a total of 148 different values of the temperatures $\Theta$ for $T=2$. For $T=8$ we used 487 values of temperatures. We used a highperformance computer which required a total of more than 94000 core hours. During the parallel simulation, we monitored equilibration by following the convergence of the statistics of $H$. The convergence takes different time scales depending on the values of $\Theta$. During the simulation we stored for $T=2$ more than 1500 configurations with corresponding values of $H$ in the range $[-7,80]$. For $T=8$ we stored about 23000 configurations with values of $H$ ranging in the interval $[-58,86]$. The configurations were spread with respect to $H$ more or less equally over the sampling intervals, but with an emphasis on the extremes of the intervals, because more values of the temperature-like parameter $\Theta$ are needed for equilibrium sampling in this range.

\section{THEORY VERSUS SIMULATIONS: SHORT TIMES}

Here we present the simulation results and compare them with the theoretical predictions from the OFM for the set of parameters (36) with $T=8$ and $L=64$. For these parameters $\epsilon=2$ is not a small number but, as was found earlier [37-39], it still corresponds to the shorttime limit. We will start from the case of $H>0$, where breaking of the mirror symmetry is expected to occur, and then proceed to the case of $H<0$ where we expect the mirror symmetry to be preserved.

\section{Large positive $H$}

In the range $10 \leq H \leq 30$, we obtained 935 independent realizations $(V, \eta)$, each allowing for an analysis of the trajectories of $h(x, \tau)$ for all values $\tau \in[0,1]$. (Note that for standard sampling, realizations exhibiting such values of $H$ would occur with their natural probabilities which are $10^{-100}$ or smaller [39], so we indeed needed to employ large-deviations algorithms to study this range of $H$.) We clearly observed the mirror symmetry breaking in the form of left- and right-traveling ramps of $h(x, \tau)$. To improve the statistics, we exploited the mutual reflection symmetry of the left- and right-traveling ramps 
with respect to each other: We flipped around $x=0$ the observed $h(x, \tau)$ profiles of the right-travelling ramp realizations and processed them together with the lefttravelling-ramps. The resulting Fig. 4 shows the average over all the simulated $h(x, \tau)$-profiles for $H \simeq 20$ at $\tau / t=0,0.35$ and 0.65 . The error bars are small, and the traveling ramp structure is very well pronounced. Figure 5 shows, at $\tau=0.5 t$, that the theoretically-predicted asymptotic optimal path [11], described by Eqs. (19)(21), is in good agreement with the simulated ramp profile. The observed discrepancies $(\lesssim 5 \%$ in terms of $H$ ) can be attributed to effects of finite $1 / H$ and $H / L$, see Eq. (33).

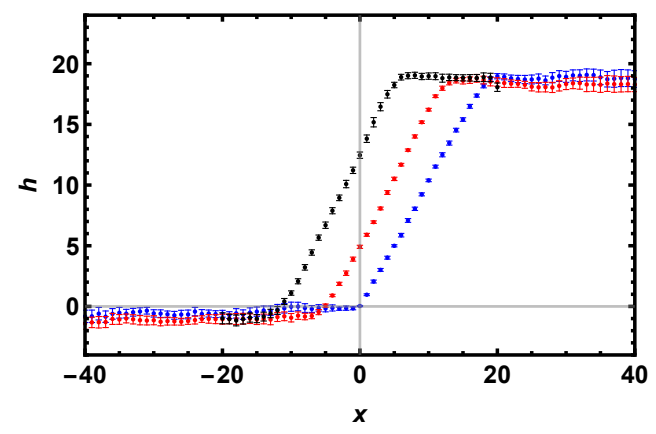

FIG. 4. The average over 9 simulated height profiles $h(x, \tau)$, conditioned on $H \simeq 20$ at rescaled times $\tau / t=0$ (blue), 0.35 (red), and 0.65 (black). The actual values of $H$ are $H \in$ $[19.95,20.1]$. Eight of these height profiles are actually righttravelling ramps flipped around $x=0$. The error bars show the standard deviation. The simulation parameters $T=8$ and $L=64$ correspond to a short time regime.

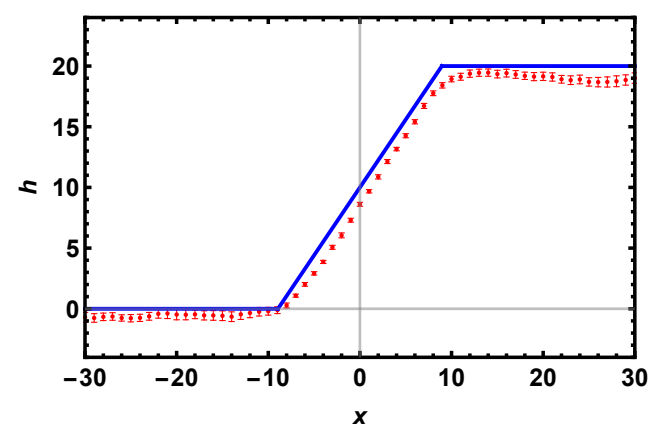

FIG. 5. A comparison, at time $\tau=0.5 t$, of the simulated average height profile conditioned on $H \simeq 20$ (see Fig. 4) with the asymptotic solution (19)-(21) for the optimal path [11].

\section{Large negative $H$}

Figure 6 shows the average over 60 simulated $h$-profiles for $H \simeq-20$ (the actual values $H \in[-20.2,-20])$. Realizations exhibiting such values of $H$ appear with natural probabilities about $10^{-450}$ or smaller [39]. Also shown is
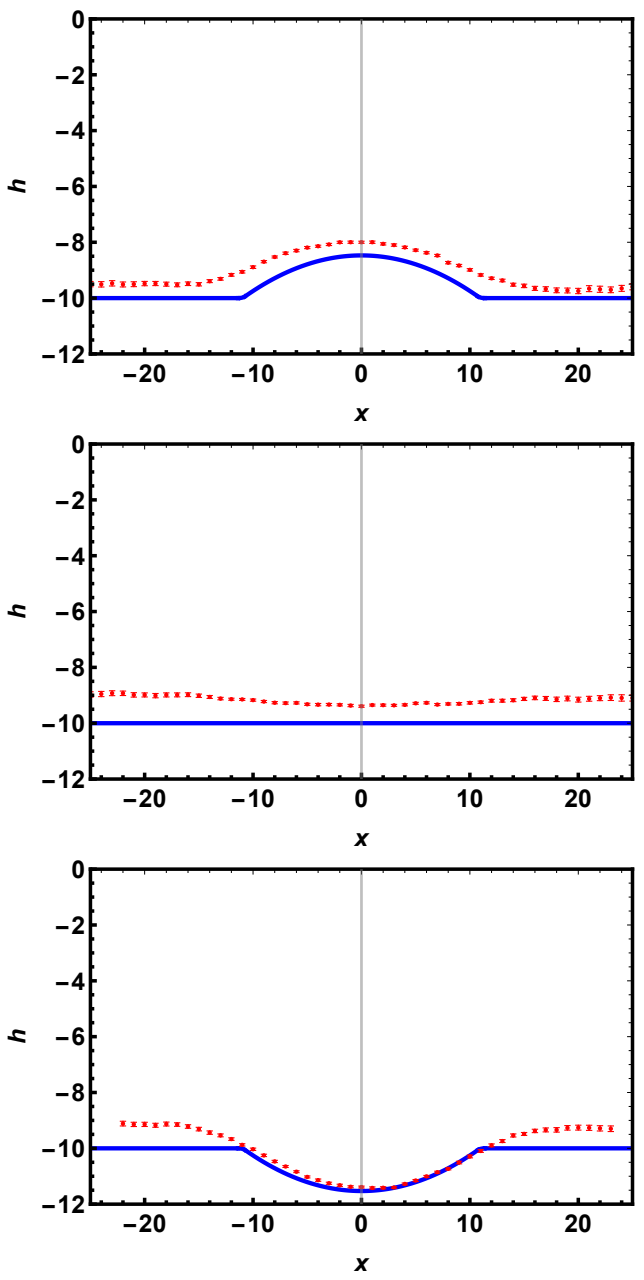

FIG. 6. A comparison of the simulated average height profile conditioned on $H \simeq-20$ (the red points with error bars) with the asymptotic solution (23)-(25) for the optimal path $h(x, \tau)$ at rescaled times $\tau / t=0.35$ (top), 0.5 (middle) and 0.65 (bottom) for $T=8$ (which corresponds to a short time) and $L=64$.

the theoretically-predicted asymptotic optimal path for $H=-20$, see Eqs. (23)-(25). The mirror symmetry is manifestly preserved here. Again, the discrepancy between the asymptotic theory and simulations is of order of $5 \%$ in terms of $|H|$, and it can be attributed to finite $|H| / L$ effects. Indeed, we also simulated the case of $H \simeq-40$ (not shown here) and found that the discrepancy between the theory (which assumes $|H| \ll L$ ) and the simulations becomes quite large.

\section{LONG TIMES}

For arbitrary values of $H$, the OFM provides an asymptotically exact large-deviation function of the KPZ height $S(H)$ see Eqs. (4) and (5), in the limit of $t \rightarrow 0$, or $\epsilon \rightarrow 0$. However, it was argued in Ref. [23], that the OFM should remain accurate in its description of (suffi- 
ciently far) distribution tails at any time $t$, or at any $\epsilon$. Since then it has been firmly established - by different methods which include rigorous mathematical proofs that, for a broad class of initial conditions, the distribution tails, as predicted by the OFM, are indeed observed at all times [53-61]. This suggests (but does not guarantee) that the dominant role of the optimal paths and their shape can be described by the OFM at long times as well, and we address this issue shortly.

Furthermore, there is an interesting difference in the structure of the lower and upper tails of $\mathcal{P}(H, t)$ at long time. This difference has been thoroughly studied (including rigorous proofs) for a class of deterministic initial conditions and, first of all, for the droplet initial condition [53-60]. The lower tail has a double structure. It consists of two distinct tails: the near and the far. The near tail, which is located at $1 \ll t^{1 / 3} \ll|H| \ll t$, scales as $-\ln \mathcal{P} \sim|H|^{3} / t$. The far tail, located at $1 \ll t \ll|H|$, exhibits the OFM scaling (7): $-\ln \mathcal{P} \sim H^{5 / 2} / \sqrt{t}$. (For brevity we dropped here the factors $\lambda, \nu$ and $D$.) An optimal path, predicted by the OFM, was indeed observed in our previous simulations of the far tail for the droplet initial condition [38], but no observations of the interface profiles corresponding to the near tail have been performed yet, for any initial condition.

The situation with the upper tail at long times is even less clear. This tail also has a near and a far regions, located at $1 \ll t^{1 / 3} \ll|H| \ll t$ and $t \ll|H|$, respectively. However, the form of the tail in these two regions turns out to be the same up to subleading corrections, and it is described by Eq. (6). For stationary initial condition this was shown in Ref. [11] by extracting the corresponding asymptotic of the Baik-Rains distribution [62] which, according to Refs. [63, 64], describes typical fluctuations of the height at long times [65]. The same situation, without exceptions, occurs for all other initial conditions considered so far (for the flat initial condition this has been known since Refs. [20, 21]). The coincidence of the near and far tails raises the question of whether a welldefined "dominating path" of the interface exists only in the far region of the upper tail, $H \gg t$, or in the near region too.

To shed light on the latter issue, we extended our numerical simulations of the interface dynamics to a lower temperature, $T=2$, keeping $L=64$. For these parameters we obtain $\epsilon=32$ which already corresponds to the long-time limit. We conducted two series of simulations: (a) conditioned on $H \simeq 20$, and (b) conditioned on $H \simeq 5$. The value of $H \simeq 20$ is not far from the regime $H>t$, and here one can expect to see an optimal path similar to that predicted by the OFM. The value of $H \simeq 5$ is already in the non-OFM regime of $t^{1 / 3}<H<t$.

Our simulation results for $T=2$ are depicted in Figs. 7 and 8 . For $H \simeq 20$ [Fig. 7 (top)] we indeed observed two optimal paths which agree fairly well with the symmetrybroken solutions - the two traveling ramps - predicted by the OFM, see Fig. 8. There are, however, some noticeable differences, clearly seen in the bottom panel of
Fig. 8. The larger noise in the profiles in the left and right plateau regions, in comparison with the simulations for $T=8$, can be explained by the larger (by a factor of 4) diffusion constant of the Brownian initial condition in this case.

Surprisingly, at $H \simeq 5$ (the bottom panel of Fig. 7), we still observed two well-defined paths with a broken mirror symmetry. Qualitatively, these "dominating paths" $h(x, \tau)$ resemble the optimal paths predicted by the OFM, but there is no quantitative agreement anymore, see Fig. 9. Still, the mere existence of two symmetry-broken dominating path of the conditioned process in this non-OFM regime is remarkable. Moreover, each of these two paths is again a mirror image of the other.
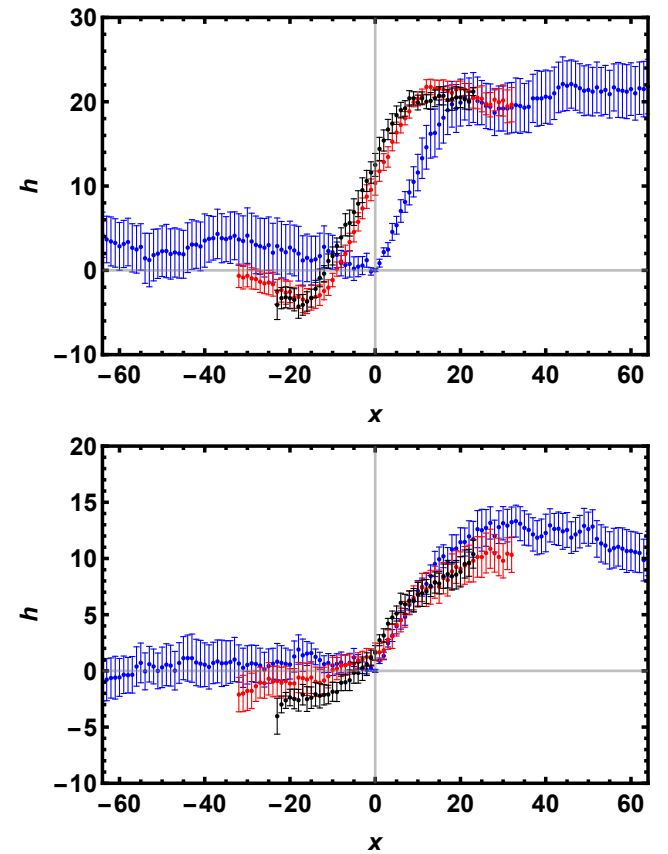

FIG. 7. The average simulated height profiles $h(x, \tau)$ conditioned on $H \simeq 20$ (top) and $H \simeq 5$ (bottom) at rescaled times $\tau / t=0$ (blue), 0.5 (red) and 0.65 (black). There were 11 measured profiles for $H \simeq 20$ and 8 profiles for $H \simeq 5$. The actual values of $H$ are $H \in[18.81,20.07]$ (top) and $H \in[4.41,5.18]$ (bottom). 5 of the height profiles, both for $H \simeq 20$ and $H \simeq 5$, were flipped around $x=0$. The error bars show the standard deviation. The simulation parameters $T=2$ and $L=64$ correspond to a long-time regime.

\section{SUMMARY AND DISCUSSION}

Optimal paths provide an instructive and fascinating characterization of a whole class of large deviations in non-equilibrium stochastic systems. By combining a mapping between the KPZ interface and the directed polymer in a random potential at high temperature with a large-deviation Monte Carlo sampling algorithm, we 

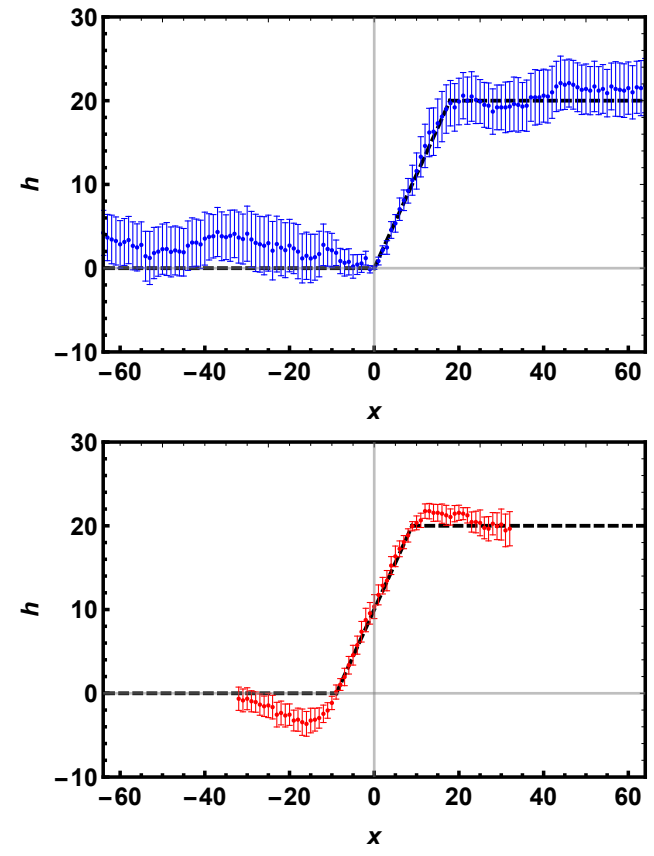

FIG. 8. A comparison of the simulated average height profiles conditioned on $H \simeq 20$ [also shown in the upper panel of Fig. 7] with the asymptotic solution (19)-(21) for the optimal path [11], shown by the dashed lines, at times $\tau / t=0$ (top) and 0.5 (bottom). The simulation parameters $T=2$ and $L=64$ correspond to a long-time regime. See Fig. 7 for other details.

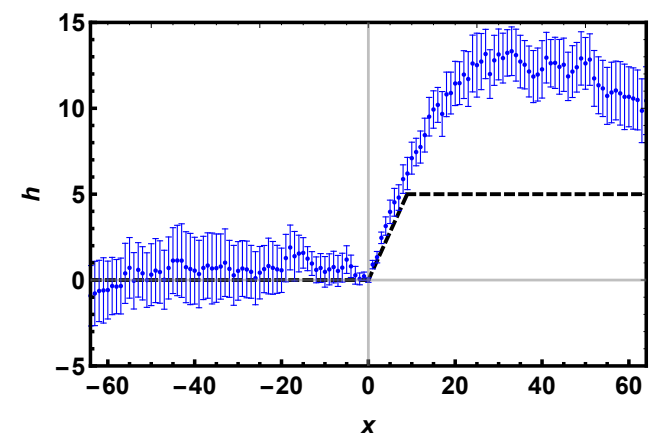

FIG. 9. The simulated average height profile conditioned on $H \simeq 5$ at long times (also shown in the upper panel of Fig. 7) and the asymptotic solution (19)-(21) for the optimal path [11] (the dashed line), at time $\tau=0$. The simulation parameters $T=2$ and $L=64$ correspond to a long-time regime. There is a clear quantitative disagreement between the OFM prediction and simulation results, but the mere presence of two well defined, mutually symmetric dominating paths of the interface (where one of them represents a mirror image of the other) is noteworthy.

were able to observe the optimal paths of the KPZ interface which determine each of the two tails of the one-point height distribution $\mathcal{P}(H, t)$ at short times, and extended the notion of the dominating path to long times. Our algorithm allowed us to probe the optimal paths which are responsible for extremely unlikely events, with probability densities down to $10^{-500}$.

In the short-time regime we clearly observed mirrorsymmetry-broken "traveling-ramp" optimal interface height profiles for the upper tail, and mirror-symmetric paths for the lower tail, in good quantitative agreement with analytical predictions from the OFM.

In the long-time regime we identified two different regions in the upper tail of $\mathcal{P}(H, t)$. In the far region (very large $H$ ) we observed an optimal path in fair agreement with the predictions of the OFM, although some noticeable differences appear. In the near region (moderately large $H$ ) the OFM is invalid. Still, we clearly observed two well-defined dominating paths of the interface. Each of them violates the mirror symmetry and represents a mirror image of the other. The presence of dominating paths of the interface hints at a possible existence of a renormalized optimal fluctuation theory for the near tails in the late-time regime of the KPZ equation.

Simulation-wise our work, alongside with Ref. [38], shows that, by using large-deviation approaches, one can not only reliably determine extremely small probabilities but also sample complete realizations from the extreme tails of corresponding distributions. This gives a valuable insight into the causes of extreme behavior of stochastic systems far from equilibrium. In the present case this is especially clear in the short-time regime, because here the OFM predicts an intimate connection between extremely small probabilities of observing very large $|H|$ and extremely rare interface configuration histories that are responsible for them. But the possibility of determining causal relations certainly also exists when no theoretical predictions are available, as was already shown earlier, e.g., in large-deviation studies of stability conditions for energy grids [47, 48]. Furthermore, even if no clear causal relations emerge, large-deviation approaches allow for a much enlarged view into correlations in complex systems.

\section{ACKNOWLEDGMENTS}

The simulations were performed in Oldenburg on the HPC cluster CARL which is funded by the DFG through its Major Research Instrumentation Programme (INST 184/157-1 FUGG) and the Ministry of Science and Culture (MWK) of the Lower Saxony State. The research of B.M. was supported by the Israel Science Foundation (grant No. 1499/20). The research of P.S. is supported by the project "High Field Initiative" (CZ.02.1.01/0.0/0.0/15_003/0000449) of the European Regional Development Fund. 
[1] G. Schütz, in "Phase Transitions and Critical Phenomena", edited by C. Domb and J. L. Lebowitz (Academic Press, London, 2001), Vol. 19.

[2] B. Derrida, J. Stat. Mech. (2007) P07023.

[3] P. I. Hurtado, C. P. Espigares, J. J. del Pozo, and P. L. Garrido, J. Stat. Phys. 154, 214 (2014).

[4] L. Bertini, A. De Sole, D. Gabrielli, G. Jona-Lasinio, and C. Landim, Rev. Mod. Phys. 87, 593 (2015).

[5] Y. Baek and Y. Kafri, J. Stat. Mech. (2015) P08026.

[6] L. Bertini, A. De Sole, D. Gabrielli, G. Jona-Lasinio, and C. Landim, Phys. Rev. Lett. 94, 030601 (2005).

[7] T. Bodineau and B. Derrida, Phys. Rev. E 72, 066110 (2005).

[8] L. Bertini, A. De Sole, D. Gabrielli, G. Jona-Lasinio, and C. Landim, J. Stat. Phys. 123, 237 (2006).

[9] P. I. Hurtado and P. L. Garrido, Phys. Rev. Lett. 107, 180601 (2011).

[10] M. Kardar, G. Parisi, and Y.-C. Zhang, Phys. Rev. Lett. 56, 889 (1986).

[11] M. Janas, A. Kamenev, and B. Meerson, Phys. Rev. E 94, 032133 (2016).

[12] N. R. Smith, A. Kamenev, and B. Meerson, Phys. Rev. E 97, 042130 (2018).

[13] The height of the evolving KPZ interface has a systematic $x$-independent component $h_{s}(t)$. For a $\delta$-correlated noise the derivative $d h_{s}(t) / d t$ is infinite. For a finite correlation length of the noise $d h_{s}(t) / d t$ approaches, at long times, a nonuniversal constant $[15,66,67]$. It is customary to get rid of this nuisance by defining $H=h(0, t)-h_{s}(t)-$ $h(0,0)$, and this is what we actually do here.

[14] J. Quastel and H. Spohn, J. Stat. Phys. 160, 965 (2015).

[15] H. Spohn, in "Stochastic Processes and Random Matrices", Lecture Notes of the Les Houches Summer School, Vol. 104, edited by G. Schehr, A. Altland, Y. V. Fyodorov, and L. F. Cugliandolo (Oxford University Press, Oxford, 2015), p. 177; arXiv:1601.00499.

[16] K. A. Takeuchi, Physica A 504, 77 (2018).

[17] Without loss of generality, we can pin the Brownian interface at the origin so that $h(x=0, \tau=0)=0$.

[18] A. Krajenbrink and P. Le Doussal, Phys. Rev. E 96, 020102(R) (2017).

[19] The one-point height distribution $\mathcal{P}(H, t)$ for the stationary KPZ interface belongs to a short list of exceptional objects for which exact representations are known. Using such a representation for the stationary interface [63, 64], Krajenbrink and Le Doussal [18] extracted the complete short-time large deviation function $S(H)$. In particular, they verified the predictions of Ref. [11] concerning the second-order DPT at $H=H_{c}$ and the two tail asymptotics (6) and (7). They also determined the value of $H_{c}$ analytically.

[20] I. V. Kolokolov and S. E. Korshunov, Phys. Rev. B 75, 140201(R) (2007).

[21] I. V. Kolokolov and S. E. Korshunov, Phys. Rev. B 78, 024206 (2008).

[22] I. V. Kolokolov and S. E. Korshunov, Phys. Rev. E 80, 031107 (2009).

[23] B. Meerson, E. Katzav, and A. Vilenkin, Phys. Rev. Lett. 116, 070601 (2016).

[24] A. Kamenev, B. Meerson, and P. V. Sasorov, Phys. Rev. E 94, 032108 (2016).
[25] B. Meerson and J. Schmidt, J. Stat. Mech. (2017) P103207.

[26] N. R Smith, B. Meerson, and P.V. Sasorov, J. Stat. Mech. (2018) 023202.

[27] B. Meerson, P. V. Sasorov, and A. Vilenkin, J. Stat. Mech. (2018) 053201.

[28] N. R. Smith, and B. Meerson, Phys. Rev. E 97, 052110 (2018).

[29] B. Meerson and A. Vilenkin, Phys. Rev. E 98, 032145 (2018).

[30] T. Asida, E. Livne, and B. Meerson, Phys. Rev. E 99, 042132 (2019).

[31] N. R. Smith, B. Meerson, and A. Vilenkin, J. Stat. Mech. (2019) 053207.

[32] Y. Lin and L. C. Tsai, Communications in Math. Phys. 386, 359 (2021).

[33] P. Y. G. Lamarre, Y. Lin and L. C. Tsai, arXiv:2106.13313.

[34] A. Krajenbrink and P. Le Doussal, Phys. Rev. Lett. 127, 064101 (2021).

[35] A. Krajenbrink and P. Le Doussal, arXiv:2107.13497.

[36] A. K. Hartmann, Big Practical Guide to Computer Simulations (World Scientific, Singapore, 2015).

[37] A. K. Hartmann, P. Le Doussal, S. N. Majumdar, A. Rosso, and G. Schehr, Europhys. Lett. 121, 67004 (2018).

[38] A. K. Hartmann, B. Meerson, and P. Sasorov, Phys. Rev. Res. 1, 032043(R) (2019).

[39] A. K. Hartmann, A. Krajenbrink, and P. Le Doussal, Phys. Rev. E 101, 012134 (2020).

[40] P. Calabrese, P. Le Doussal, and A. Rosso, Europhys. Lett. 90, 20002 (2010).

[41] J. M. Hammersley and K. W. Morton, Math. Proc. Cambr. Phil. Soc 52, 449 (1956).

[42] A. K. Hartmann, Phys. Rev. E 65, 056102 (2002).

[43] A. K. Hartmann, Eur. Phys. J. B 84, 627-634 (2011).

[44] A. K. Hartmann and M. Mézard, Phys. Rev. E 97, 032128 (2018).

[45] A. K. Hartmann, S. Majumdar, and A. Rosso, Phys. Rev. E 88, 022119 (2013).

[46] G. Claussen, A. K. Hartmann, and S. N. Majumdar, Phys. Rev. E 91, 052104 (2015).

[47] T. Dewenter and A.K. Hartmann, New J. Phys 17, 015005 (2015).

[48] Y. Feld and A. K. Hartmann, Chaos 29, 113103 (2019).

[49] S. Wolfsheimer, B. Burghardt, and A. K. Hartmann, Algor. Mol. Biol. 2, 2 (2007).

[50] A. K. Hartmann, Phys. Rev. E 89, 052103 (2014).

[51] W. Staffeldt and A. K. Hartmann, Phys. Rev. E 100, 062301 (2019).

[52] M. E. J. Newman and G. T. Barkema, Monte Carlo Methods in Statistical Physics (Clarendon Press, Oxford, 1999).

[53] P. Le Doussal, S. N. Majumdar, and G. Schehr, Europhys. Lett. 113, 60004 (2016).

[54] P. Sasorov, B. Meerson, and S. Prolhac, J. Stat. Mech. 063203 (2017).

[55] I. Corwin, P. Ghosal, A. Krajenbrink, P. Le Doussal, and L. C. Tsai, Phys. Rev. Lett. 121, 060201 (2018).

[56] L. C. Tsai, arXiv:1809.03410. 
[57] A. Krajenbrink, P. Le Doussal, and S. Prolhac, Nucl. Phys. B 936239 (2018).

[58] I. Corwin and P. Ghosal, Electron. J. Probab. 25, 66 (2020).

[59] P. Le Doussal, J. Stat. Mech. 043201 (2020).

[60] P. Ghosal and Y. Lin, arXiv:2007.06505.

[61] P. L. Ferrari and B. Vető, Electron. Commun. Probab. 26, 15 (2021).

[62] J. Baik and E. M. Rains, J. Stat. Phys. 100, 523 (2000).

[63] T. Imamura and T. Sasamoto, Phys. Rev. Lett. 108, 190603 (2012); J. Stat. Phys. 150, 908 (2013).
[64] A. Borodin, I. Corwin, P. L. Ferrari, and B. Vető, Math. Phys. Anal.; Geom. 18, 1 (2015).

[65] Imamura and Sasamoto [63] and Borodin et al. [64] obtained an explicit exact representation for $\mathcal{P}(H, T)$ in terms of Fredholm determinants. They also proved that, at long times and for typical fluctuations of $H, \mathcal{P}(H, t)$ converges to the Baik-Rains distribution [62].

[66] T. Gueudré, P. Le Doussal, A. Rosso, A. Henry, and P. Calabrese, Phys. Rev. E 86, 041151 (2012).

[67] M. Hairer, Annals of Math. 178, 559 (2013). 